\title{
INVESTIGACIÓN
}

Recibido: 12/03/2021 --- Aceptado: 15/04/2021 --- Publicado: 24/05/2021

\section{PERCEPCIÓN DE SABORES DE MALTEADAS A TRAVÉS DE CANALES SENSORIALES POR MEDIO DE ELECTROENCEFALOGRAFÍA Y GALVANOMETRÍA}

\section{Perception of milkshakes flavors through sensory channels by means of electroencephalography and galvanometry}

Héctor Orlando Valenzuela Reinoso. Universidad Autónoma de Occidente. Colombia.

hovalenzuela@uao.edu.co

Stephania Aristizabal Aristizabal. Universidad Autónoma de Occidente. Colombia. stephania.aristizaba@uao.edu.co

Natalia Díaz Ariza. Universidad Autónoma de Occidente. Colombia

Natalia.diaz_ariza@uao.edu.co

\section{Cómo citar el artículo:}

Valenzuela Reinoso, H, O., Aristizabal Aristizabal, S., y Díaz Ariza, N. (2021). Percepción de sabores de malteadas a través de canales sensoriales por medio de electroencefalografía y galvanometría. Vivat Academia. Revista de Comunicación, 154, 285-300. http://doi.org/10.15178/va.2021.154.e1352

\section{RESUMEN}

Debido a los avances en las neurociencias, la evolución y accesibilidad a dispositivos biométricos, que permiten la captura de respuestas fisiológicas ante estímulos externos, se ha presentado una forma diferente de conocer al consumidor y poder adaptar todas las estrategias de mercadeo lo más personalizado posible en una disciplina llamada Neuromarketing. Atendiendo a esta realidad, el objetivo de la presente investigación es analizar de forma experimental la percepción frente a ciertos estímulos sensoriales derivados de cinco sabores (vainilla, fresa, chocolate, chicle y cookies and cream) de malteadas pertenecientes a la industria de helados y postres. Se realizó una muestra de 20 sujetos, seleccionados mediante una entrevista semiestructurada para garantizar que no habían consumido los sabores de esas malteadas. Posteriormente, fueron expuestos a los cinco sabores en tres momentos diferentes, sólo con el olfato como primer momento, sólo con el canal sensorial del gusto y, por último, sólo con el canal sensorial de la vista. Esto nos permitió capturar la actividad cerebral de ondas (theta, alpha, beta y hi-beta), y también la actividad 
Valenzuela Reinoso, H, O., Aristizabal Aristizabal, S., y Díaz Ariza, N.

Percepción de sabores de malteadas a través de canales sensoriales por medio de electroencefalografía y galvanometría.

galvánica, mediante un electroencefalograma (EEG) y un medidor (GSR). Los resultados permitieron observar que los sabores de chocolate y fresa son los que mostraron más cambios y diferencias de género, así mismo, el canal del olfato es el que mayor incidencia tiene en la forma como se perciben los sabores.

PALABRAS CLAVE: Neuromarketing - electroencefalograma - respuesta galvánica de la piel - percepción - comportamiento del consumidor - sabores de malteadas ondas cerebrales.

\section{ABSTRACT}

Due to the advances in neuroscience, the evolution and the accessibility of biometric devices, it is possible to capture physiological responses caused by external stimulus, thanks to this a different way to know the consumer has been presented in order to adapt marketing strategies and to make them as personalized as possible in a discipline called Neuromarketing. Realizing this, the aim of this research is to experimentally analyze the perception of certain sensory stimulus derived from five shake flavors (vanilla, strawberry, chocolate, gum and cookies and cream), flavors that we commonly find in the dessert and ice cream industry. We applied a semistructured interview to find out some people who have never tasted those shake flavors, the final sample was made of 20 people. Subsequently, the 20 people were exposed to the five flavors at three different times. only with smell as the first moment, only with the sensory channel of taste and, finally, only with the sensory channel of sight. This made possible to capture brain wave activity (theta, alpha, beta and hi-beta), as well as galvanic activity, using an electroencephalogram (EEG) and a meter (GSR). The results showed that the chocolate and strawberry flavors evidenced the most changes and gender differences, likewise, the smell channel is the one that has the greatest impact on the way flavors are perceived.

KEYWORDS: Neuromarketing - Electroencephalogram - Galvanic Skin Response Perception - Behavior Consumer - Flavors Milkshakes - Brain Waves.

\section{INTRODUCCIÓN}

El objetivo del presente trabajo es investigar el comportamiento de los individuos desde el ámbito de la neurociencia, lo que nos permitirá tener una comprensión más completa y objetiva de la percepción de los sujetos al momento de experimentar los cinco estímulos de malteadas (vainilla, fresa, chocolate, chicle y cookies and cream). Lo cual, fue gracias a la utilización de dos instrumentos biométricos proporcionadas por el Neuromarketing, el Electroencefalograma (EEG), llamado Neurobit Optima de 4 canales y el medidor de respuesta galvánica (GSR). De igual manera, este trabajo busca ayudar a presentar lineamientos en el área del marketing sensorial, que se ha convertido en los últimos años en una herramienta con un gran potencial para analizar e investigar de una manera más eficiente y eficaz el comportamiento del consumidor en el momento de adquirir algún bien o servicio en particular. El marketing sensorial se define como un medio óptimo y científico para analizar los 
Valenzuela Reinoso, H, O., Aristizabal Aristizabal, S., y Díaz Ariza, N.

Percepción de sabores de malteadas a través de canales sensoriales por medio de electroencefalografía y galvanometría.

diferentes estímulos y elementos que los consumidores perciben a través de los sentidos, generando ciertas atmósferas que permite a los individuos interpretar de manera coherente y significativa su entorno según sus experiencias vividas, gustos y preferencias particulares (Gómez y García, 2012), por lo tanto, el mercado actual exige una constante innovación en estrategias para atraer y construir relaciones reales con los clientes, y no basta solo con realizar cambios en factores como el precio o imagen. Esta innovación puede desarrollarse por medio de la neurociencia del consumidor que "permite una comprensión más completa y objetiva de los deseos y acciones del consumidor, y se convierte en una herramienta versátil y consistente en el acompañamiento de las empresas para el ajuste y realización de sus estrategias de mercado" (Salazar, 2012). Por lo cual, el neuromarketing al ser una disciplina que analiza el comportamiento humano desde un enfoque comercial combina "el conocimiento psicológico del comportamiento, economía y neurociencia del consumidor, adaptando teorías y métodos de la neurociencia para identificar correlaciones del comportamiento del sujeto" (Núñez- Cansado, et al., 2020). Así mismo, De la Morena en su tesis doctoral hace énfasis en el gran aporte realizado por los instrumentos de medición del Neuromarketing para el estudio científico y preciso de las variables que componen el comportamiento de consumo de los individuos en un ambiente comercial, mencionando el EEG como una de las técnicas del neuroimagen más precisas del mercado, el cual "ha registrado el aumento del nivel de atención, agrado, emociones o motivación cuando se genera una necesidad o deseo en la demanda de los consumidores mejorando las actuales técnicas de investigación de mercado del marketing tradicional" (De la Morena, 2016).

Un acercamiento a los estudios de neurociencias aplicados al marketing sensorial, permiten contextualizar la importancia de investigar en esta área del mercadeo. Los estudios realizados por Martín a través de neuroimagen nos permiten aclarar que:

Existe una diferencia dinámica neuronal de los olores agradables y desagradables. Los primeros activan principalmente el área olfativa de los lóbulos frontales, en especial del hemisferio derecho; sin embargo, los olores desagradables inciden sobre la amígdala (parte fundamental en las emociones) y la corteza de la ínsula del lóbulo temporal. En ambos casos las señales llegan desde el bulbo olfatorio a los centros cerebrales donde se desencadenan y regulan las emociones y los recuerdos que asocian sensación-emoción (Martín, 2013).

Estos hallazgos nos permiten estructurar el diseño metodológico de la investigación para ubicar los electrodos del EEG en el lóbulo prefrontal de los sujetos de estudio. Por otro lado, en una investigación con base en imágenes de resonancia magnética (fMRI) entre hombres y mujeres, se encontró "que los participantes varones tratan la calidad del producto como una constante y tienden a apoyar la teoría de la calidad original. Sin embargo, las participantes femeninas mostraron una activación cerebral diferenciable a través de tres factores, sugiriendo una representación dinámica para la calidad del producto" (Yi-Ting y Ming-Sung, 2018). Entonces, estas diferencias de género también pueden llegar afectar cualquier decisión de consumo, ya que: 
Valenzuela Reinoso, H, O., Aristizabal Aristizabal, S., y Díaz Ariza, N.

Percepción de sabores de malteadas a través de canales sensoriales por medio de electroencefalografía y galvanometría.

Cualquier cosa que logre afectar de manera negativa el sentido del olfato, produce directamente una afectación a la capacidad propia de percibir los sabores, generando un rechazo, pues solo a través de un sabor se pueden evocar recuerdos, emociones y sentimientos del pasado, ya que toda la información generada y a su vez transmitida a la corteza cerebral pasa también al sistema límbico (Según Manzano, et al., 2012).

En ese orden de ideas, Sepúlveda en su estudio de marketing sensorial, afirma que:

La percepción de la presencia de un aroma es un proceso relativamente lento. Frente a los 45 milisegundos que tardamos en percatarnos visualmente de un objeto, necesitamos diez veces más tiempo, 450 milisegundos, para detectar un olor. Esto se debe a que las neuronas olfatorias no están mielinizadas, lo que significa que la información debe viajar de neurona a neurona, por lo que se ralentiza el proceso. La lentitud con la que se detecta un olor se repite en el tiempo que tarda en abandonarnos. Las sensaciones olfatorias se desvanecen con lentitud, dependiendo de la temperatura y del flujo de aire, por lo que en ocasiones se tiene la impresión de que el aroma es casi pegajoso. (Sepúlveda, 2017).

Por lo cual, entender la conexión entre el olfato y las emociones será el éxito de las estrategias de mercado olfativas para las empresas, debido a que ayudará a comprender su potencial como herramienta para despertar afectos, pasiones o sentimiento que perduren en el tiempo. Más adelante, contempla dos cualidades de los aromas en el marketing olfativo, el placer y la congruencia, en donde agrega "el placer, recoge la experiencia intrínseca del olor, mientras que la congruencia conecta el olor con el contexto: tienda, producto o marca" (Sepúlveda, 2017).

Del mismo modo, Sanz (2016) de la Universidad de Valladolid de España, apoya el supuesto del gran poder que posee el olfato como herramienta efectiva en la comunicación y ambientación en el punto de venta, además de ser la mejor forma de crear experiencias emocionales que se posicionen en el "Top of Mind" y el "Top of Heart" de los consumidores, en donde a través de diferentes evaluaciones de empresas que han implementado estrategias olfativas, se ha podido observar un aumento en la facturación de hasta un $40 \%$. Señala también dos aspectos importantes en el marketing olfativo, en primer lugar, el olfato es el único de los sentidos capaz de saltarse cualquier barrera para inhabilitar su función. Adicionalmente, menciona la capacidad de los olores para quedar almacenados en la memoria del consumidor durante un tiempo mucho mayor que el sentido más utilizado en la publicidad actual, la vista. Por tanto, el sentido del olfato al ser utilizado con menos frecuencia debido a la ignorancia de su utilidad y su gran poder invisible en la mente de los consumidores se ha posicionado como un sentido potencial para la implementación de estrategias especializadas y efectivas con bases científicas.

De igual manera, Gómez (2012) realizó un trabajo de investigación con el objetivo de obtener una visión más clara de la identidad olfativa de una marca a través de una 
Valenzuela Reinoso, H, O., Aristizabal Aristizabal, S., y Díaz Ariza, N.

Percepción de sabores de malteadas a través de canales sensoriales por medio de electroencefalografía y galvanometría.

estrategia invisible y silenciosa. Este trabajo relata, en relación con el sentido del olfato, la creciente utilización del marketing olfativo para el desarrollo de estrategias cada vez más especializadas con el fin de incrementar la percepción de valor entre marca y consumidor, de esta manera, dicha estrategia permite un reconocimiento de marca anclado a unas de las funciones más primitivas y poderosas de los individuos: el olfato y su conexión profunda con la memoria emocional. Menciona, además, la importancia de la aplicación de odotipos en productos y en puntos de venta de consumo, para generar asociaciones fuertes entre un aroma específico y una experiencia que, gracias a la memoria, se revive una y otra vez al percibir dicho aroma. Estos odotipos, se refieren a "una forma aromática estable que se inscribe como uno de los elementos distintivos para una marca". (Sánchez, et al., 2012).

No obstante, la medición eléctrica cerebral no es la única prioridad de investigación en el Neuromarketing, debido a que el tacto, al ser el sentido más extenso en los seres humanos, cuenta con una gran cantidad de terminaciones nerviosas a las que se les nombra "receptores del tacto, estos se encuentran en la capa más externa de la piel y son los encargados de transportar las sensaciones hacia el cerebro a través de las fibras nerviosas" (Suárez, 2012). Por lo cual, deduce que la piel se ha convertido en un factor importante a la hora de medir el comportamiento de compra de los individuos. Actualmente, existe una herramienta especializada llamada respuesta galvánica, conocida como GSR, la cual sirve para medir la actividad galvánica de la piel en tiempo real, por lo que cada cambio emocional que presente un individuo como: el miedo, la ansiedad, el estrés o la alegría, logran causar una variación relevante en la conductividad eléctrica y con ello un impacto directo en su proceso de toma de decisión.

En cuanto a la elección de alimentos, a menudo los consumidores basan su proceso de toma de decisión de forma inconsciente. Dicha toma de decisión está influenciada por un complejo conjunto de emociones, sentimientos, actitudes y valores que son imposibles de evaluar simplemente preguntando a los consumidores sus opiniones (Stasi, et al, 2018). De hecho, las técnicas tradicionales, como los auto informes o las entrevistas, permiten principalmente la medición de reacciones conscientes y racionales a un producto o publicidad. En un estudio se investigó utilizando herramientas de evaluación sensorial, la capacidad predictiva sobre la disposición a pagar, usando escalas de Likert y escalas de intensidad, se tuvo en cuenta así mismo si la calidad extrínseca o intrínseca ejerce un impacto similar en la disposición a pagar del consumidor (Gallardo, et al., 2018). Los resultados de este estudio demuestran que la información recopilada sobre la preferencia por un atributo de calidad sensorial tiene una mejor capacidad predictiva para la disposición a pagar, en comparación con la información sobre la percepción de la intensidad percibida del atributo de calidad sensorial.

En este contexto, las hipótesis planteadas en esta investigación son las siguientes:

1. Existen diferencias en la actividad eléctrica entre hombres y mujeres para cada uno los sabores (vainilla, fresa, chocolate, chicle y cookies and cream). 
Valenzuela Reinoso, H, O., Aristizabal Aristizabal, S., y Díaz Ariza, N.

Percepción de sabores de malteadas a través de canales sensoriales por medio de electroencefalografía y galvanometría.

2. Existen diferencias entre hombres y mujeres para los canales sensoriales utilizados (olfato, gusto y vista)

\section{OBJETIVOS}

\subsection{Objetivo general}

Conocer la percepción de sabores (vainilla, fresa, chocolate, chicle y cookies and cream) por medio de canales sensoriales (vista, olfato y gusto) a través de dispositivos biométricos como el electroencefalograma y el medidor de respuesta galvánica.

\subsubsection{Objetivo específico 1.}

Capturar las respuestas fisiológicas en los canales sensoriales de la vista, el gusto, el olfato y el tacto, frente a sabores de malteadas (vainilla, fresa, chocolate, chicle y cookies and cream).

\subsubsection{Objetivo específico 2 .}

Encontrar las diferencias entre hombres y mujeres de acuerdo a los canales sensoriales de la vista, el gusto, el olfato y el tacto, frente a sabores de malteadas (vainilla, fresa, chocolate, chicle y cookies and cream).

\subsubsection{Objetivo específico 3 .}

Evaluar las diferencias entre hombres y mujeres, sobre la percepción de los sabores (vainilla, fresa, chocolate, chicle y cookies and cream).

\section{METODOLOGÍA}

Con el objetivo de llevar a cabo esta investigación, se usó un encefalograma llamado Neurobit Optima de 4 canales (Imagen 1) y un software para la captura de ondas cerebrales y galvánicas de la piel llamado BioExplorer (Imagen 2). Con este dispositivo se capturaron las diferentes ondas cerebrales (Alpha - Theta - Beta - HiBeta), de los sujetos de estudio al ser expuestos a uno de los cinco estímulos de sabores elegidos. Las ondas cerebrales según Martín (2013), se asocia de esta manera: La onda Alpha, está relacionada con el engagement, es decir, la presencia de esta onda está asociada con estados de compromiso y conexión con el estímulo al que está siendo expuesto, la onda Theta, está relacionada con relajación, es decir, que cuando se presenta esta onda, el estímulo al que está siendo expuesto le genera tranquilidad, por otro lado, la onda Beta se relaciona con estados alerta, similar al proceso que experimentan los sujetos con la onda Hi-beta, la cual se relaciona con un estado de alerta en un nivel más alto. 
Valenzuela Reinoso, H, O., Aristizabal Aristizabal, S., y Díaz Ariza, N.

Percepción de sabores de malteadas a través de canales sensoriales por medio de electroencefalografía y galvanometría.

El proceso de muestreo aleatorio se basó en seleccionar a 20 sujetos (10 hombres y 10 mujeres) que no hubiesen probado los sabores de la marca que fue seleccionada, debido a que podían presentar algún tipo de sesgo. Dicha población, consistió en personas entre los 18 a 25 años de edad. Los experimentos tuvieron una duración aproximada de 30 minutos por persona, los cuales se llevaron a cabo en la cámara de Gesell de la Universidad Autónoma de Occidente, ya que este lugar permitió aislar estímulos externos, logrando maximizar la calidad del proceso investigativo.

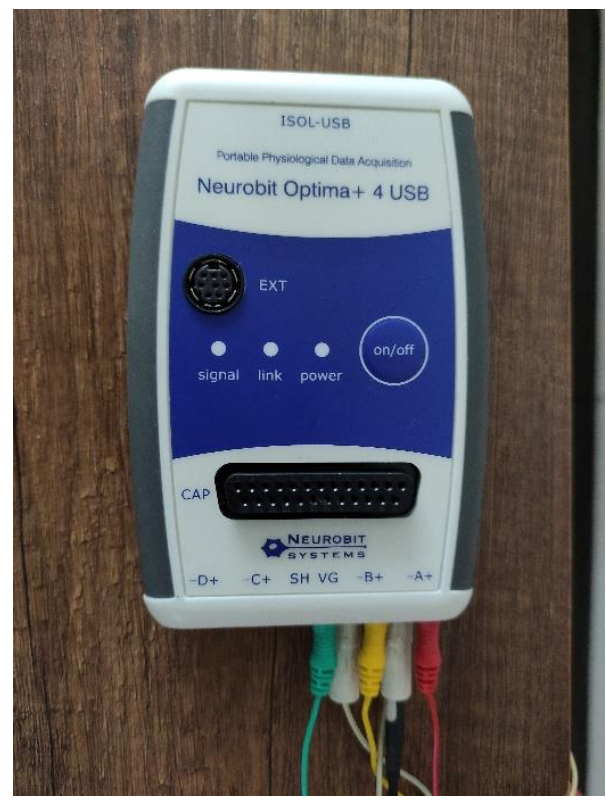

Imagen 1: Electroencefalograma Optima 4 canales.

Fuente: elaboración propia.

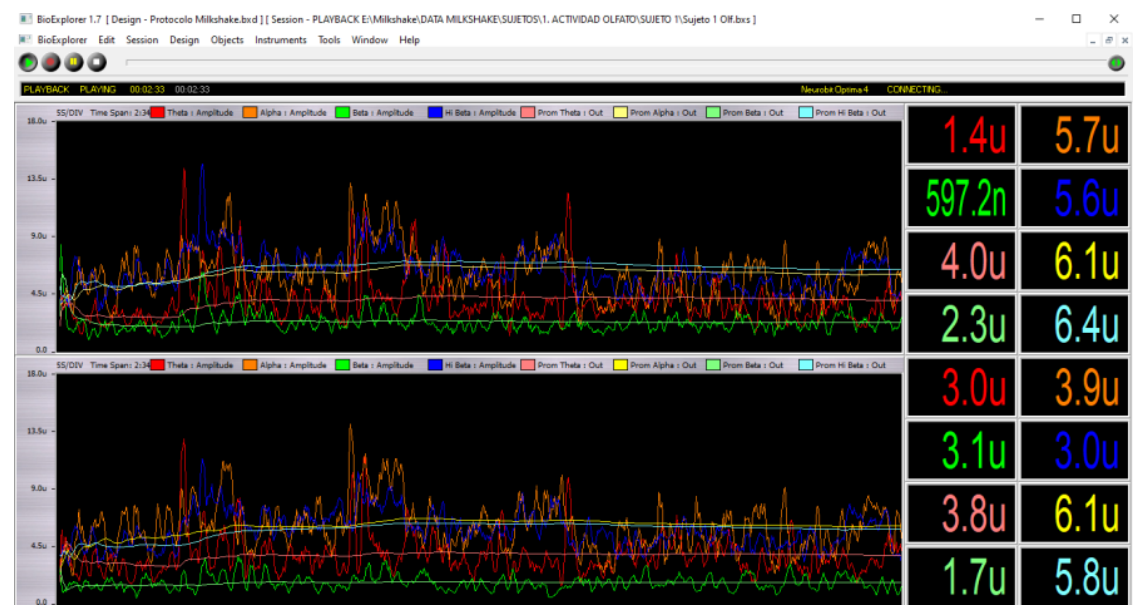

Imagen 1: Software Bioexplorer.

Fuente: Elaboración propia.

Después de que la persona era seleccionada, se conectaban al EEG ajustando los electrodos en posición PF1 y PF2 (Imagen 3), según el sistema 10-20 para ajustar electrodos. Estos electrodos se ubicaron en la corteza prefrontal izquierda y derecha. 
Valenzuela Reinoso, H, O., Aristizabal Aristizabal, S., y Díaz Ariza, N.

Percepción de sabores de malteadas a través de canales sensoriales por medio de electroencefalografía y galvanometría.

El electrodo de referencia se ubicó en CZ, según el sistema 10-20 para ajustar electrodos, y finalmente, un electrodo en la oreja, el cual tiene la función de ser el polo a tierra. Por último, se midió el nivel de impedancia para garantizar la calidad de la medición electroencefalográfica. Se eligió la zona prefrontal del lóbulo frontal porque según Martín (2013) “aporta todo lo que nos hace verdaderamente humanos: la elaboración del yo, la conciencia de nosotros mismos, de nuestras emociones y de nuestro entorno. Es el asiento de la percepción consciente, donde se encuentra el libre albedrío, nuestros pensamientos y nuestra capacidad para aprender, razonar y racionalizar" (p. 75). Por lo que en la zona prefrontal es donde se realiza el proceso de toma de decisión de los individuos participantes del experimento, es decir, todo el proceso de percepción termina con la decisión de elegir o no un sabor, por lo tanto, medir esta zona del cerebro nos permite determinar que sabor elegirá en un punto de venta. Por otro lado, el GSR registra las ondas a través de 3 electrodos (Imagen 4), los cuales se conectan en los dedos índice, corazón y anular (actúa como polo a tierra), de la mano derecha de cada individuo, y son capturadas las ondas de la piel, las cuales, al igual que con el EEG, quedan registradas en el software BioExplorer, para posteriormente ser analizadas por cada individuo, y lograr identificar su respuesta ante los diferentes estímulos presentados en el momento del experimento.

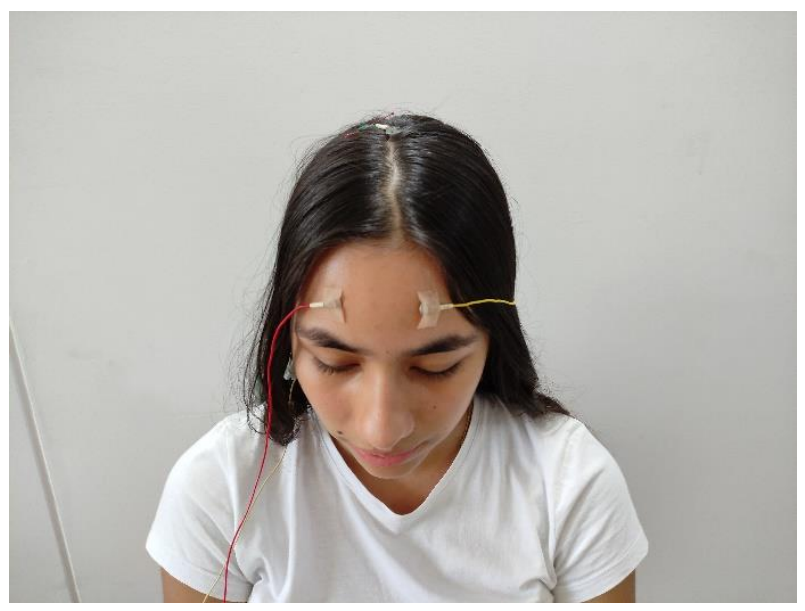

Imagen 3: Ubicación de electrodos en PF1 y PF2.

Fuente: Elaboración propia.

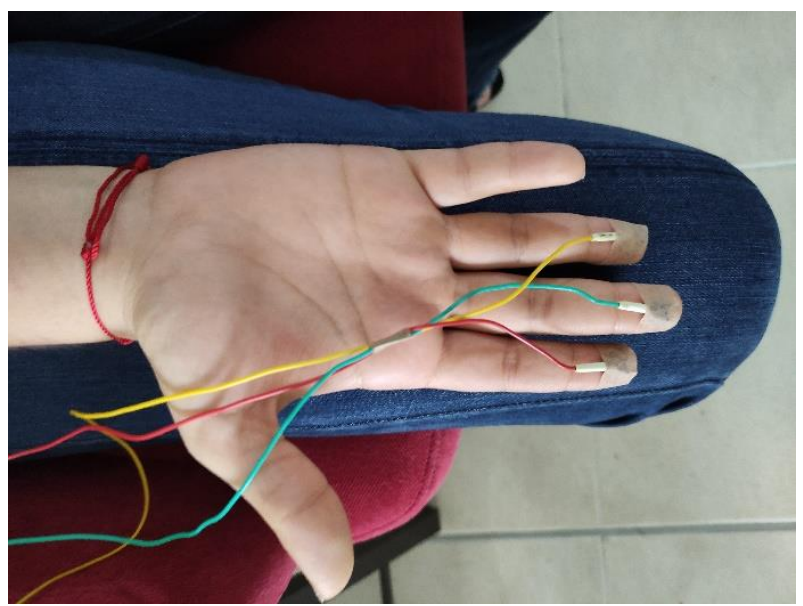


Valenzuela Reinoso, H, O., Aristizabal Aristizabal, S., y Díaz Ariza, N.

Percepción de sabores de malteadas a través de canales sensoriales por medio de electroencefalografía y galvanometría.

Imagen 4: Ubicación de electrodos para captura de respuesta galvánica.

Fuente: Elaboración propia.

Siguiente al ajuste de los electrodos y conexión exitosa en los sujetos, se prosigue a iniciar la medición del EEG y el GSR en tiempo real, donde se prepararon cinco vasos con los sabores y fueron presentados en el siguiente orden: 1. Vainilla, 2. Fresa, 3. Chocolate, 4. Chicle y 5. Cookies \& Cream (Imagen 5). Cada uno de estos sabores se presentaron con una latencia de 20 segundos para eliminar cualquier rastro del sabor anterior. Una vez pasado los 20 primeros segundos, se presentaba el primer estímulo (vainilla) por 10 segundos, luego se esperaba 20 segundos sin ningún estímulo para continuar con el segundo estímulo y así sucesivamente, hasta acabar con la presentación de los cinco estímulos.

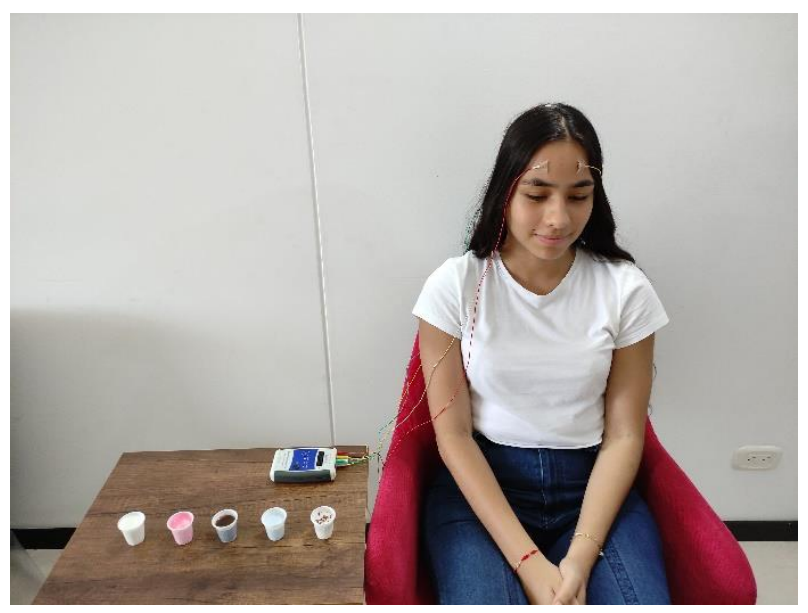

Imagen 5: Sabores y montaje listo para el experimento.

Fuente: Elaboración propia.

El experimento se llevó a cabo en tres momentos diferentes utilizando siempre la cámara de Gesell. En el momento 1, los sujetos tuvieron los ojos tapados, en donde solo experimentaron los olores de los cinco estímulos, mientras se medía la actividad eléctrica del cerebro y de la piel. En el momento 2, los sujetos tuvieron la nariz tapada y los ojos vendados para así solo probar los sabores y medir las respuestas fisiológicas. Finalmente, en el momento 3, se llevó a cabo la última medición de los sujetos, donde a través de una pantalla se visualizaban las malteadas hechas a base del sabor seleccionado y midiendo las respuestas fisiológicas.

En cuanto a la forma de analizar los datos es importante mencionar, que cada ser humano es único en su estructura neuronal, esto se debe a que la potencia de sus ondas varía entre cada individuo, por lo que compararlas entre sí sería contraproducente. En este orden de ideas, la presente investigación busca identificar el momento en que la onda cambia, para así poder estandarizar dichas variaciones y otorgarles rangos de valor. De esta manera, se aplicó un condicional lógico para determinar tres condicionales: el condicional 1 (bajo), refleja que la onda se encuentra 
Valenzuela Reinoso, H, O., Aristizabal Aristizabal, S., y Díaz Ariza, N.

Percepción de sabores de malteadas a través de canales sensoriales por medio de electroencefalografía y galvanometría.

por debajo de cero, es decir, la onda no subió y el sujeto no demostró cambios ante el estímulo de manera contundente. Por otro lado, el condicional 2 (medio), muestra que los datos son mayores a cero, pero no están por encima de la desviación estándar, por lo que la onda subió de manera parcial y no reacciona fuertemente ante el estímulo, el condicional 3 (alto), evidencia que la onda está por encima de la desviación estándar, lo que quiere decir que además de subir, lo hizo por fuera de los rangos normales. Por último, los datos se analizaron en el programa SPSS, usando el estadístico Chi Cuadrado para establecer la relación existente entre las variables estudiadas, y de esta manera dar cumplimiento a los objetivos específicos planteados.

Es muy importante resaltar que en esta investigación solo se tomaron los datos más relevantes, es decir, los que tienen un nivel de significancia menor o igual al 5\%. Por lo cual, los datos que están por encima de este porcentaje, no se tomaron en cuenta para ser analizados en la investigación, pues no se consideran relevantes.

Finalmente, la variable dependiente es la respuesta fisiológica tanto cerebral como galvánica y la variable independiente son los sabores que se eligieron para el experimento.

\section{RESULTADOS}

El cerebro humano es complejo tanto en su estructura como en su funcionamiento, por lo que existe una diversidad de estudios que exploran cómo funciona el pensamiento, siendo un sistema unificado y flexible en el tiempo. La Dr. Benzinger en un estudio, menciona que el cerebro a pesar de tener unas divisiones especializadas, actúa como un todo en un sistema interdependiente, es decir, el pensamiento humano y su capacidad de actuar racionalmente se lleva a cabo en la corteza cerebral, sin embargo, el sistema reptiliano y límbico fueron los encargados de captar los estímulos e información del exterior de acuerdo a experiencias pasadas del sujeto, donde seguidamente pasan a la corteza para ser "procesadas y codificadas, más específicamente, por el lóbulo prefrontal; por lo que se dice que primero sentimos y luego pensamos" (Martín, 2013). Como consecuencia, para poder comprender como funciona el pensamiento humano, se enfoca en la corteza al dividirla en "cuatro áreas de igual tamaño conformadas por dos fisuras transversales: la fisura central y la fisura longitudinal" (Benzinger, 2002). Dichas áreas especializadas, presentan características diferentes para poder funcionar de manera eficiente, por lo que postuló el "modelo de dominancia" para poder explicar la dinámica interna del cerebro, donde exalta que el ser humano nace con "un modo claramente preferido, en el que tenemos una velocidad y una eficiencia naturales e increíbles, y tres modos no preferidos, cada uno de los cuales nos resulta más difícil debido a que es notablemente más lento y menos eficiente" (Benzinger, 2002). En este orden de ideas, cuando alguna área de la corteza cerebral trabaja en una frecuencia baja, el cerebro necesita menos esfuerzo, gastando menos energía y logrando realizar la tarea de manera eficiente y efectiva. 
Valenzuela Reinoso, H, O., Aristizabal Aristizabal, S., y Díaz Ariza, N.

Percepción de sabores de malteadas a través de canales sensoriales por medio de electroencefalografía y galvanometría.

El análisis de la presente investigación, parte de la utilización de técnicas de Neuromarketing como el uso del encefalograma (mide la actividad eléctrica del cerebro) y el galvanómetro (mide la actividad eléctrica de la piel), instrumentos de medición usados para evaluar los fenómenos cognitivos y emocionales que se evidencian en el proceso de toma de decisión de los consumidores en cuanto a la elección de malteadas. Por lo que se pudo capturar la actividad cerebral en microvoltios de cada onda antes, durante y después de cada uno de los cinco sabores de malteadas. Los resultados arrojados por el EEG y el GSR, fueron analizados cuantitativamente con el programa Statistical Package for the Social Sciences (SPSS), usando el estadístico chi cuadrado para establecer la relación existente entre las variables estudiadas. De esta manera, se busca dar respuesta a la primera hipótesis planteada, la cual menciona que se quiere conocer si existen diferencias en la actividad eléctrica entre hombres y mujeres para cada uno los sabores; dicha hipótesis se puede confirmar con la evidencia en la tabla 1, la cual muestra los resultados esperados. Es interesante encontrar que las mujeres presentaron estados de alerta cuando experimentaron los sabores de chocolate y fresa, es decir, al momento que baja la actividad cerebral, este requiere menos esfuerzo, lo que se traduce en un menor consumo de energía y una mayor comodidad con el estímulo experimentado. De manera diferente, los hombres presentaron una actividad cerebral baja con los mismos sabores que fueron encontrados en los resultados de las mujeres (chocolate y fresa). Sin embargo, dichos sabores causaron estados de relajación en el género masculino.

Tabla 1: Género vs sabor-onda

\begin{tabular}{|c|c|c|c|c|c|c|c|c|c|c|c|c|c|c|c|c|}
\hline \multirow{3}{*}{ Sabores } & \multicolumn{8}{|c|}{ Mujeres } & \multicolumn{8}{|c|}{ Hombres } \\
\hline & \multicolumn{4}{|c|}{ Canal A } & \multicolumn{4}{|c|}{ Canal B } & \multicolumn{4}{|c|}{ Canal A } & \multicolumn{4}{|c|}{ Canal B } \\
\hline & $\Theta$ & $\alpha$ & $\beta$ & $\begin{array}{c}\mathrm{H} \\
\beta\end{array}$ & $\Theta$ & a & $\beta$ & $\mathrm{H} \beta$ & $\Theta$ & a & $\beta$ & $\mathrm{H} \beta$ & $\Theta$ & $a$ & $\beta$ & $\mathrm{H} \beta$ \\
\hline Vainilla & & & & & & & & & & & & & & & & \\
\hline Fresa & & & & & & & $\begin{array}{l}0,01 \\
4\end{array}$ & & & & & & 0,012 & & & \\
\hline Chocolate & & & $\begin{array}{l}0,00 \\
8\end{array}$ & & & & & & 0,049 & & & & 0,031 & & & \\
\hline Chicle & & & & & & & & & & & & & & & & \\
\hline Cookies & & & & & & & & & & & & & & & & \\
\hline
\end{tabular}

Fuente: Esta tabla resume los sabores con los resultados más significativos de género vs sabores, exponiendo los niveles de significancia correspondientes a cada variable.

Elaborado con datos propios en el software de IBM SPSS Statistics Visor.

La hipótesis 2 (Existen diferencias entre hombres y mujeres para los canales sensoriales utilizados "olfato, gusto y vista") fue analizada de manera conjunta con la hipótesis 1 (Existen diferencias en la actividad eléctrica entre hombres y mujeres para cada uno los sabores "vainilla, fresa, chocolate, cookies and cream y chicle"), en 
Valenzuela Reinoso, H, O., Aristizabal Aristizabal, S., y Díaz Ariza, N.

Percepción de sabores de malteadas a través de canales sensoriales por medio de electroencefalografia y galvanometría.

donde ambas se lograron por medio del uso del estadístico chi-cuadrado, tomando como base la variable género, ambas hipótesis se pudieron aceptar.

En cuanto a los resultados del género vs sentido, la tabla 2 aborda el sentido del olfato, en este, es posible apreciar que las mujeres al experimentar los olores de fresa y chocolate presentaron estados de alerta, dicho estado es asociado a un menor esfuerzo de energía a la hora de percibir los estímulos. Por otro lado, los hombres continúan presentando estados de relajación, al momento de experimentar el estímulo de chocolate.

Tabla 2: Género vs sentido (olfato)

\begin{tabular}{|c|c|c|c|c|c|c|c|c|c|c|c|c|c|c|c|c|}
\hline \multirow{3}{*}{ Sabores } & \multicolumn{8}{|c|}{ Mujeres } & \multicolumn{8}{|c|}{ Hombres } \\
\hline & \multicolumn{4}{|c|}{ Canal A } & \multicolumn{4}{|c|}{ Canal B } & \multicolumn{4}{|c|}{ Canal A } & \multicolumn{4}{|c|}{ Canal B } \\
\hline & $\Theta$ & a & $\beta$ & $\mathrm{H} \beta$ & $\Theta$ & $a$ & $\beta$ & $\mathrm{H} \beta$ & $\Theta$ & $a$ & $\beta$ & $\mathrm{H} \beta$ & $\Theta$ & $\mathrm{a}$ & $\beta$ & $\mathrm{H} \beta$ \\
\hline Vainilla & & & & & & & & & & & & & & & & \\
\hline Fresa & & & & & & & 0,025 & & & & & & & & & \\
\hline Chocolate & & & $\begin{array}{l}0,02 \\
5\end{array}$ & & & & & & & & & & 0,007 & & & \\
\hline Chicle & & & & & & & & & & & & & & & & \\
\hline Cookies & & & & & & & & & & & & & & & & \\
\hline
\end{tabular}

Fuente: Esta tabla resume los sabores con los resultados más significativos de género vs sentido del olfato, exponiendo los niveles de significancia correspondientes a cada variable. Elaborado con datos propios en el software de IBM SPSS Statistics Visor.

La tabla 3 evidencia que los hombres al experimentar los estímulos, el sabor de chicle fue el único que creo estados de engagement (compromiso). Al contrario, las mujeres no presentaron resultados significativos en el sentido del gusto en cuanto a los sabores experimentados.

Tabla 3: Género vs sentido (gusto)

\begin{tabular}{|c|c|c|c|c|c|c|c|c|c|c|c|c|c|c|c|c|}
\hline \multirow{3}{*}{ Sabores } & \multicolumn{8}{|c|}{ Mujeres } & \multicolumn{8}{|c|}{ Hombres } \\
\hline & \multicolumn{4}{|c|}{ Canal A } & \multicolumn{4}{|c|}{ Canal B } & \multicolumn{4}{|c|}{ Canal A } & \multicolumn{4}{|c|}{ Canal B } \\
\hline & $\Theta$ & $a$ & $\beta$ & $\mathrm{H} \beta$ & $\Theta$ & $a$ & $\beta$ & $\mathrm{H} \beta$ & $\Theta$ & $a$ & B & $\mathrm{H} \beta$ & $\Theta$ & $a$ & $\beta$ & $\mathrm{H} \beta$ \\
\hline Vainilla & & & & & & & & & & & & & & & & \\
\hline Fresa & & & & & & & & & & & & & & & & \\
\hline Chocolat & & & & & & & & & & & & & & & & \\
\hline Chicle & & & & & & & & & & & & & & 0,001 & & \\
\hline Cookies & & & & & & & & & & & & & & & & \\
\hline
\end{tabular}

Fuente: Esta tabla resume los sabores con los resultados más significativos de género vs sentido del gusto, exponiendo los niveles de significancia correspondientes a cada variable. Elaborado con datos propios en el software de IBM SPSS Statistics Visor. 
Valenzuela Reinoso, H, O., Aristizabal Aristizabal, S., y Díaz Ariza, N.

Percepción de sabores de malteadas a través de canales sensoriales por medio de electroencefalografía y galvanometría.

Finalmente, la tabla 4 expone los resultados del sentido de la vista, donde las mujeres presentaron un resultado significativo con el estímulo visual de chicle, creando estados de alerta en los sujetos. Por otro lado, los hombres mostraron unos resultados significativos más diversos en cuanto a estímulos visuales de las malteadas, ya que se observa que tuvieron una frecuencia cerebral baja con los estímulos de vainilla, fresa y cookies \& cream, logrando estados de relajación (Theta).

Tabla 4: Género vs sentido (vista)

\begin{tabular}{|c|c|c|c|c|c|c|c|c|c|c|c|c|c|c|c|c|}
\hline \multirow{3}{*}{ Sabores } & \multicolumn{8}{|c|}{ Mujeres } & \multicolumn{8}{|c|}{ Hombres } \\
\hline & \multicolumn{4}{|c|}{ Canal A } & \multicolumn{4}{|c|}{ Canal B } & \multicolumn{4}{|c|}{ Canal A } & \multicolumn{4}{|c|}{ Canal B } \\
\hline & $\Theta$ & $a$ & $\beta$ & $\mathrm{H} \beta$ & $\Theta$ & $a$ & $\beta$ & $\mathrm{H} \beta$ & $\Theta$ & $a$ & $B$ & $\mathrm{H} \beta$ & $\Theta$ & $a$ & $\beta$ & $\mathrm{H} \beta$ \\
\hline Vainilla & & & & & & & & & 0,024 & & & & & & & \\
\hline Fresa & & & & & & & & & 0,047 & & & & 0,024 & & & \\
\hline Chocolate & & & & & & & & & & & & & & & & \\
\hline Chicle & & & 0,005 & & & & & & & & & & & & & \\
\hline Cookies & & & & & & & & & 0,047 & & & & & & & \\
\hline
\end{tabular}

Fuente: Esta tabla resume los sabores con los resultados más significativos de género vs sentido de la vista, exponiendo los niveles de significancia correspondientes a cada variable. Elaborado con datos propios en el software de IBM SPSS Statistics

\section{DISCUSIÓN} Visor.

El Neuromarketing ha logrado ser una gran herramienta de estudio para diferentes investigaciones científicas en el área del mercadeo, capaz de lograr analizar con mayor precisión el comportamiento de los individuos a nivel neuronal. Sin embargo, una vez concluida la investigación y al haber realizado de cerca una investigación experimental con la herramienta del Neuromarketing, es preciso reconocer el alcance y las limitaciones que tuvo la misma.

En la investigación se logró demostrar que los sabores de chocolate y fresa generaron en las mujeres una sensación de alerta y en los hombres un estado de relajación, estos resultados se lograron a través de herramientas de Neuromarketing, que nos permitieron lograr un alcance científico y preciso, esto con el fin de elaborar un diseño metodológico que permitiera conocer las diferencias de género en la percepción de múltiples estímulos.

Por otra parte, en cuanto a las limitaciones, específicamente en la medición experimental que se realizó del sentido del olfato, en el momento que las personas dejaban de oler la malteada, se les debió haber dado el estímulo olfativo de café, esto con el fin de neutralizar los diferentes olores de las malteadas, logrando así que el 
Valenzuela Reinoso, H, O., Aristizabal Aristizabal, S., y Díaz Ariza, N.

Percepción de sabores de malteadas a través de canales sensoriales por medio de electroencefalografía y galvanometría.

siguiente olor lo asimilarán de la mejor manera, y los datos arrojarán un resultado con mayor exactitud en el momento de proceder a analizarlos.

Otra de las limitaciones fue la falta de tiempo en la investigación, debido a que se presentaron inconvenientes con los sujetos al momento de conectarlos al encefalograma (EEG), ya que en ocasiones presentaban una estructura ósea muy gruesa y afectaba de manera directa la medición óptima de su actividad cerebral. Por ende, dichos sujetos eran descartados $\mathrm{y}$, en consecuencia, se perdía tiempo importante en la búsqueda de nuevos sujetos que cumplieran con los requisitos de la investigación, atrasando el cronograma ya establecido.

En cuanto a la percepción de género, fue interesante corroborar las diferencias cerebrales que existen entre hombres y mujeres, lo cual influencia de manera contundente su comportamiento de compra. Para las mujeres, los datos sugieren que existe un posible patrón de respuesta en su actividad eléctrica para los sabores de chocolate y fresa. En otras palabras, el EEG y GSR, arrojan la frecuencia más baja en la onda beta con dichos sabores, en el sentido del olfato generando estados de alerta en los sujetos. Por otro lado, los hombres demuestran un patrón de respuesta direccionada la onda Theta, experimentando estados de relajación para los sentidos de la vista y el olfato, sin embargo, para el sentido del gusto lo hombres fueron los únicos en presentar resultado significativo, evidenciando que se encontraban en un estado de compromiso (onda alpha) a la hora de probar el sabor de chicle.

\section{REFERENCIAS}

Benziger, K. (2002). Maximizando: El Potencial de Sus Talentos. ISBN: 1-880931-11-7. THRT.

De la Morena, A. (2016). Neuromarketing y nuevas estrategias de la mercadotecnia: análisis de la eficiencia publicitaria en la diferenciación de género y la influencia del marketing sensorial y experiencial en la decisión de compra. Complutense de Madrid. https://eprints.ucm.es/id/eprint/38701/

Gallardo, R., Karina, H., Yeon A., Silva-Jaimes, M., \& Flores-Orozco, J. (2018). Investigating consumer food choice behavior: an application combining sensory evaluation and experimental auctions. Ciencia e investigación agraria,45(1), 110. http://dx.doi.org/10.7764/rcia.v45i1.1765

Gómez Ramírez, C. (2012). La identidad olfativa: una estrategia invisible y silenciosa. ISSN-0124-5821.

http://revistavirtual.ucn.edu.co/index.php/RevistaUCN/article/view/392

Gómez, M. y García, C. (2012). Marketing sensorial: Cómo desarrollar la atmósfera del establecimiento comercial. Distribución y consumo, 39(2), 30-39. ISSN 1132-0176. https://www.mercasa.es/media/publicaciones/196/1336046531_pag_030040_Gomez.pdf. 
Valenzuela Reinoso, H, O., Aristizabal Aristizabal, S., y Díaz Ariza, N.

Percepción de sabores de malteadas a través de canales sensoriales por medio de electroencefalografía y galvanometría.

Hsu, M. Y. -T. and Cheng, J. M. -S. (2018). fMRI neuromarketing and consumer learning theory: Word-of-mouth effectiveness after product harm crisis. European Journal of Marketing, 52(1/2), 199-223. https:// doi.org/10.1108/EJM-12-2016-0866

Malhotra, N. (2008). Investigación de mercados (5a. ed.). ISBN:9786073235600. Pearson educación.

Manzano, R., Gavilán, D., Avello, M., Abril, C. y Serra, T. (2012). Marketing Sensorial. Comunicar con los sentidos en el punto de venta. ISBN-13:978-8483228128. Prentice Hall.

Martín, L. (2013). Neurociencia, empresa y marketing. ISBN: 978-84-16462-42-1. ESIC.

Núñez-Cansado, M., López López, A., y Caldevilla Domínguez, D. (2020). Situation of Neuromarketing Consulting in Spain. Front. Psychol. 11:1854. https://doi.org/10.3389/fpsyg.2020.01854

Salazar, C. (2012). La neurociencia del consumidor como horizonte de investigación, conceptos y aplicaciones. Revista Universidad y Empresa, 14(21), 143-166. ISSN: 0124-4639. https://www.redalyc.org/pdf/1872/187222420007.pdf

Sánchez-Herrera, J. y Pintado Blanco, T. (2012). Nuevas tendencias en Comunicación. ISBN: 9788417129354. Editorial ESIC.

Sanz, I. (2016). El marketing sensorial en las tiendas de moda. Universidad de Valladolid. (Trabajo final de grado). https://core.ac.uk/download/pdf/211100499.pdf

Sepúlveda, J. (2017). Marketing sensorial como estrategia de mercados en almacenes de ropa, pp. 26-27. Universidad de Ciencias Aplicadas y Ambientales. https://repository.udca.edu.co/bitstream/11158/906/1/MONOGRAFIA\%20JEN NIFER\%20SEP\%C3\%9ALVEDA.pdf.

Stasi, A., Songa, G., Mauri, M., Ciceri, A., Diotallevi, F., Nardone, G. y Russo, V. (2018). Neuromarketing empirical approaches and food choice: A systematic review, 650-664. https://doi.org/10.1016/j.foodres.2017.11.049 .

Suárez, M. (2012). Neuromarketing: una forma de romper los paradigmas del marketing convencional. Universidad del Rosario. https://repository.urosario.edu.co/handle/10336/4016 
Valenzuela Reinoso, H, O., Aristizabal Aristizabal, S., y Díaz Ariza, N.

Percepción de sabores de malteadas a través de canales sensoriales por medio de electroencefalografía y galvanometría.

\section{AUTORES}

\section{Héctor Orlando Valenzuela Reinoso}

El Mg Orlando es profesor del Departamento de Mercadeo y Negocios Internacionales de la Universidad Autónoma de Occidente de Colombia. Posee títulos de Mágister en Administración de Negocios (MBA), especialista en Formulación y evaluación de proyectos y Neuropsicología. Sus campos de investigación son el comportamiento del consumidor, Neuromarketing y la investigación de mercados.

ORCID ID: https:// orcid.org/0000-0001-9713-8011

\section{Natalia Díaz Ariza}

Estudiante del programa Mercadeo y Negocios Internacionales de la Universidad Autónoma de Occidente de Colombia. Realizó su tesis de pregrado en neuromarketing con lo cual se puede realizar el artículo en cuestión.

\section{Stephania Aristizábal Aristizábal}

Estudiante del programa Mercadeo y Negocios Internacionales de la Universidad Autónoma de Occidente de Colombia. Realizó su tesis de pregrado en neuromarketing con lo cual se puede realizar el artículo en cuestión. 